Research Article

\title{
Optimization Design Strategy of Electroadhesive Devices with Interdigital Electrodes Based on the Multiparameters Theoretical Model
}

\author{
Shuang Lai $\mathbb{D}^{1},{ }^{1}$ Jintao Zhang $\mathbb{D}{ }^{2},{ }^{2}$ Yaxuan Yan $\mathbb{D D}^{2}$ and Huahua Yu $\mathbb{D}^{1}$ \\ ${ }^{1}$ College of Engineering, Nanjing Agricultural University, Nanjing 210031, China \\ ${ }^{2}$ College of Artificial Intelligence, Nanjing Agricultural University, Nanjing 210031, China
}

Correspondence should be addressed to Shuang Lai; 32218220@njau.edu.cn

Received 10 August 2021; Accepted 3 September 2021; Published 15 September 2021

Academic Editor: Qiuqin Sun

Copyright (c) 2021 Shuang Lai et al. This is an open access article distributed under the Creative Commons Attribution License, which permits unrestricted use, distribution, and reproduction in any medium, provided the original work is properly cited.

Electroadhesion is an adhesion mechanism applying high voltage to generate adhesive force. The electroadhesion system can generate and maintain adhesive force on almost any object, solving the challenge of handling irregular and rough surface objects as well as fragile objects. The electroadhesive pad is a key component of the electroadhesion system for interacting with the target object. By optimizing the design of the electroadhesive pad, the electroadhesion system provides greater adhesive force and achieves better adhesion. In this study, a multiparameter theoretical model including the dimensional parameters of the electroadhesive pad has been developed and an optimization design strategy for specific applications has been proposed. By considering both the key parameters influencing the electroadhesive force and the practical constraints of equipment and materials, this strategy allows the optimization design methods of electroadhesive pads to be further extended to applications. The influence of each parameter on the optimization results has been evaluated by calculating and comparing the optimized values under different conditions, and it has been demonstrated that the size of the pad also has an effect on the optimized values. A 3D simulation model has been established to simulate the effect of electroadhesion, and the accuracy of the optimization results has been verified by comparing the theoretical and simulation results. An application example has been performed and the results have shown that the structure of the electroadhesive pad can be optimized by using this strategy, thus maximizing the generated electroadhesive force and improving the overall performance of the electroadhesion system.

\section{Introduction}

Electroadhesion is the electrostatic attraction force that occurs between the electroadhesive device and the contacting material when a high voltage is applied [1]. Compared with other adhesion mechanisms, electroadhesion usually exhibits a lot of advantages, such as the properties of lightweight and high compliance due to its flexible film structure [2]. In addition, the noncontact attraction force can be produced by electroadhesive devices, thus enhancing the adaptability to various materials, such as uneven and porous materials $[3,4]$. Electroadhesion can also be applied in different environments and is particularly promising for space applications [5]. Moreover, electroadhesion is electrically controllable and can be actuated by simply turning on the high-voltage DC power, which reduces the complexity of the system and the difficulty of operation [6]. Furthermore, electroadhesion is considered an ultra-low energy consumption technology [7], as it usually acts as a capacitor.

As a result of these advantages, electroadhesion has been extensively employed in diverse fields, such as wafer transportation and processing in the semiconductor industry [8], fabric gripping in clothing manufacturing $[9,10]$, an adhesion mechanism for climbing robots [11-14], electroadhesive grippers for holding workpieces [15-17], and end effectors for conducting space missions [18].

The components of an electroadhesion system typically include an electroadhesive pad, a high-voltage DC power supply, a control device, and a substrate being manipulated [19]. Among these parts, the electroadhesive pad is the 
critical one to generate the adhesive force and interact with the object being adhered or picked up. Generally, the pad consists of the electrodes encapsulated within the insulating material, and the electrodes are composed of a periodic array of electrode strips. The dielectric material is utilized to insulate the electrode from the external environment to prevent charge neutralization and to increase the breakdown voltage of the pad. When a high voltage is applied to the electrode, charges are generated on the target object, resulting in electroadhesive force. However, the charge generation principle is different for the conductor and the nonconductor; the former is generated by electrostatic induction of charges on the electrodes and the latter is generated by the polarization effect of the electric field between the electrodes.

Based on the structural characters of the electroadhesive pad and the generation mechanism of electrostatic force, considerable research efforts have been devoted to studying the interaction mechanism between the pad and target objects. For instance, to calculate the electroadhesive force for the general case, an analytical model was proposed by Bo and Guo [20]. The model is applicable to solids with variable dielectric properties and surface roughness and can be used to optimize the design of electroadhesive pads. The relationship between several key parameters, including applied voltage, relative dielectric constant, and thickness of the insulating material, and geometry of the electrode and electroadhesive force was investigated by Guo et al. [21] through theoretical model calculations and simulation analysis. Moreover, numerous design optimization methods have been further proposed to improve the performance of electroadhesive devices based on the relationship between the influencing parameters and the electroadhesive force. For example, to improve the electroadhesive force, the method of increasing the relative permittivity by doping the dielectric layer with highly polarized materials was proposed by Mahmoudzadeh et al. [22]. Based on the finite element analysis (FEA) method, two-dimensional simulation and three-dimensional simulation were used by Bigharaz et al. [23] to optimize the electrode width, spacing, and geometry. It was observed that the sine-wave shaped electrode could enhance the obtainable electroadhesive force and thus improve the electroadhesive pad performance. Kim et al. [24] developed an electrode shape optimization design method and concluded that there exists an optimal ratio of electrode width to spacing that maximizes the electroadhesive force applied to the nonconductor. However, the effect of the overall size of the electroadhesive pad on the electroadhesive force is rarely considered in these optimization design approaches, and the optimization design of application-specific electroadhesive pads remains a challenge. In the study of Choi et al. [25], a quantitative relationship between the lifting force and the total length of the electrode boundary edge was determined and a model equation for the electrostatic lifting force including the electrode boundary length parameter was presented. However, this equation was obtained by fitting the experimental results and lacked a basis for theoretical derivation. To implement the optimization design of electroadhesive pads for different cases,
West et al. [26] introduced a general design optimization method using submillimeter parameters. In addition, Wang et al. [27] established an overall flow diagram based on parametric analysis to select the optimal design parameters for electroadhesive pads. Although these works have attempted to provide strategies for optimizing the geometry of electroadhesive pads, they ignore practical influences such as the limitations of power supply devices and manufacturing equipment.

To derive the theoretical relationship between electroadhesive pad size and electroadhesive force, a multiparameter theoretical model containing the electroadhesive pad size parameters has been established in this paper. To further extend the optimization design method to practical applications, an optimization design strategy based on the multiparameter theoretical model has been proposed by considering the key design parameters (size, structure, and material parameters of the electroadhesive pad) and the actual constraints (available materials, limitations of manufacturing processes, and power supply equipment). The remainder of this paper has been organized as follows: in Section 2, a multiparameter theoretical model has been developed by using the parallel plate capacitance model. In Section 3, an optimization design strategy for the application-specific electroadhesive pad has been presented. In Section 4, the accuracy of the theoretical model and the feasibility of the design strategy have been verified by calculating and comparing the theoretical and simulation results. Finally, the conclusions have been summarized in Section 5.

\section{Principle and Theoretical Model of Electroadhesion}

The typical electroadhesive pad depicted in Figure 1(a) consists of conductive electrodes and dielectric material, which are tightly bonded together. The structure of interdigital electrodes is shown in the front view, the width of the electrodes is defined as $T_{w}$, and the spacing between the electrodes is presented by the symbol $T_{s}$. The dimensional parameters of the pad are also illustrated in Figure 1(a), including the pad length $L_{M}$ and the pad width $L_{N}$. In addition, as shown in the cross-sectional view, the thickness of the insulation layer close to the target object is defined as $t$. Furthermore, to facilitate the analysis and calculation of the electroadhesive force, in view of the periodicity and symmetry of the pad, the area within the red frame shown in Figure 1(a) is chosen as a unit.

2.1. Electroadhesive Force of the Conductor. As shown in Figure 1(b), when the power supply is turned on, charges of opposite polarity are generated on the conductor surface by the attraction of the charges generated on the electrode surface. The electrodes and the conductor are separated by an insulating layer that prevents the migration and neutralization of charges on their surfaces. Consequently, an electric field is created between the electrodes and the conductor surfaces, turning the whole system into a capacitor [28]. As shown in Figure 1(b), the equivalent 
capacitors are connected in parallel with an equal number of electrodes. Based on the parallel plate capacitor model, the sum electrostatic force between the pad and the conductor surface can be calculated by

$$
F_{z}=\frac{1}{2} \frac{C^{2} U^{2}}{\varepsilon_{0} S}=\frac{1}{2} \varepsilon_{0} S\left(\frac{U}{t / \varepsilon_{r 1}}\right)^{2}
$$

where $U$ is the applied voltage, $t$ is the thickness of the insulation layer, $\varepsilon_{0}$ is the absolute dielectric constant of vacuum $\left(8.85 * 10^{-12} \mathrm{~F} / \mathrm{m}\right), \varepsilon_{r 1}$ is the relative permittivity of the insulation layer, and $S$ denotes the facing area or effective area of the capacitor plate.

According to the principle of electrostatic induction, the effective area is the same as the area of the electrodes. Considering the size of the pad and the structure of the electrodes, the electrode area can be expressed as

$$
S=\frac{L_{M} * T_{w} *\left(T_{s}+L_{N}\right)}{\left(T_{w}+T_{s}\right)} .
$$

2.2. Electroadhesive Force of the Nonconductor. When the electroadhesive device is used to hold nonconductive objects, alternating positive and negative polarity voltages are required to be applied to the electrodes. Therefore, when the electrodes are energized by a high-voltage power supply, a strong electric field will be generated between the adjacent electrodes due to the electric potential difference. Under the action of the electric field, polarized charges will be produced on nearby objects and eventually lead to electrostatic attraction between the electroadhesive device and objects.

To simplify the calculation of the field strength, the electric field between adjacent electrodes is replaced by the electric field distributed according to concentric electric field lines as shown in Figure 2 [29]. According to the shape character of the concentric electric field, its electric field strength can be represented by

$$
E=\frac{U}{\pi T}
$$

where $T$ corresponds to the radius of the concentric circle and $U$ is the potential difference between the electrodes.

Considering the structural features of the electroadhesive pad, the number of units can be given as

$$
n=\frac{L_{M}}{T_{w}+T_{s}} .
$$

In addition, by integrating the field strength of a unit and substituting equation (3), the total electric field strength generated in the unit is derived as

$$
E^{\prime}=L * \int_{0.5 T_{s}}^{0.5\left(T_{w}+T_{s}\right)} E \mathrm{~d} T=L * \frac{U}{\pi} * \ln \left(1+\frac{T_{w}}{T_{s}}\right)
$$

where $L=L_{N}-T_{w}$ is the length of the region in the unit with concentric circular electric fields distributed, $0.5 T_{s}$ is the minimum radius, and $0.5\left(T_{w}+T_{s}\right)$ is the maximum radius of the field.

Based on the polarization principle, the dielectric is polarized under the effect of this electric field, and the number of generated polarized charges can be calculated as

$$
Q=\varepsilon_{0} \varepsilon_{\mathrm{eff}} * E^{\prime}
$$

where $\varepsilon_{\text {eff }}$ is the relative effective dielectric constant of the dielectric region through which the electric field passes.

From the concentric circle-shaped electric field model, both the dielectric layer and the nonconductor within the electric field are polarized, and thus the entire electric field region from the positive electrode through the dielectric layer and the object to the negative electrode can be equated to the capacitance shown in Figure 2. Then, according to the phenomenon of interlayer polarization and the principle of calculating series capacitance, the relative permittivity of the equivalent capacitance can be obtained as

$$
\varepsilon_{\mathrm{eff}}=\frac{\pi \varepsilon_{r 1} \varepsilon_{r 2}\left(T_{s}+0.5 T_{w}\right)}{4 \varepsilon_{r 2} t+\varepsilon_{r 1}\left[\pi\left(T_{s}+0.5 T_{w}\right)-4 t\right]},
$$

where, to simplify the operation, the average radius value $T_{s}+0.5 T_{w}$ of the semicircular electric field is taken for the calculation of the spacing between the two plates of the equivalent capacitance (Guo et al., [29]).

According to the electrostriction theory, the electroadhesive force generated between the capacitance can be expressed as

$$
f_{z}=\frac{1}{2} \frac{Q^{2}}{\varepsilon_{0} S}
$$

where $S$ is the area of the parallel plate of the equivalent capacitance in a unit and can be expressed by $S=0.5 T_{w} * L$.

In view of equations (4)-(7) and (8), the sum electroadhesive force of the whole pad can be described by

$$
F_{z}=n * f_{z}=\frac{L_{M}}{T_{w}+T_{s}} * \frac{L_{N}-T_{w}}{T_{w}} * \varepsilon_{0} * \varepsilon_{\mathrm{eff}}^{2} *\left[\frac{U}{\pi} * \ln \left(1+\frac{T_{w}}{T_{s}}\right)\right]^{2}
$$

According to this multiparameter theoretical model, the electroadhesive force generated in the whole pad is proportional to the pad length $\left(L_{M}\right)$ and applied voltage $(V)$ and shows a positive correlation with the relative permittivity $\left(\varepsilon_{r 1}\right.$ and $\left.\varepsilon_{r 2}\right)$ as well as the pad width $\left(L_{N}\right)$, and it is negatively related to the thickness of the dielectric layer $(t)$. Moreover, there is an optimal electrode width $\left(T_{w}\right)$ that can maximize the force. 


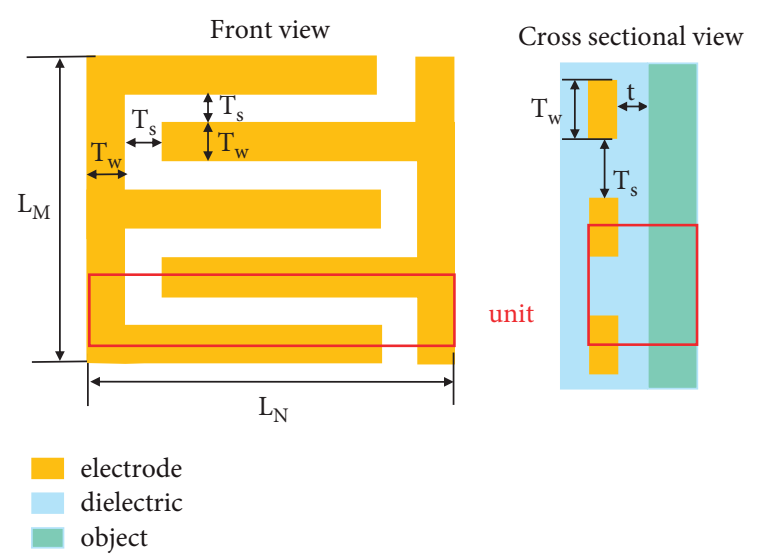

(a)

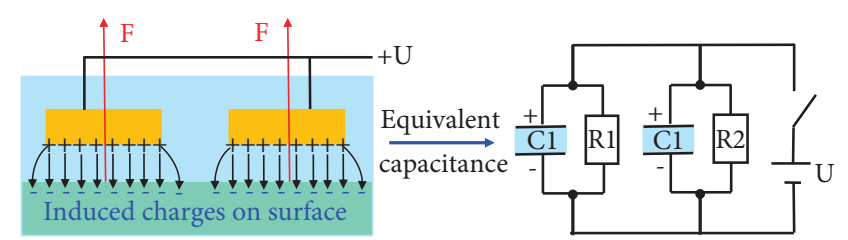

(b)

FIgURE 1: (a) Structure of the electroadhesive pad. (b) Electrostatic adhesion principle of the conductor.

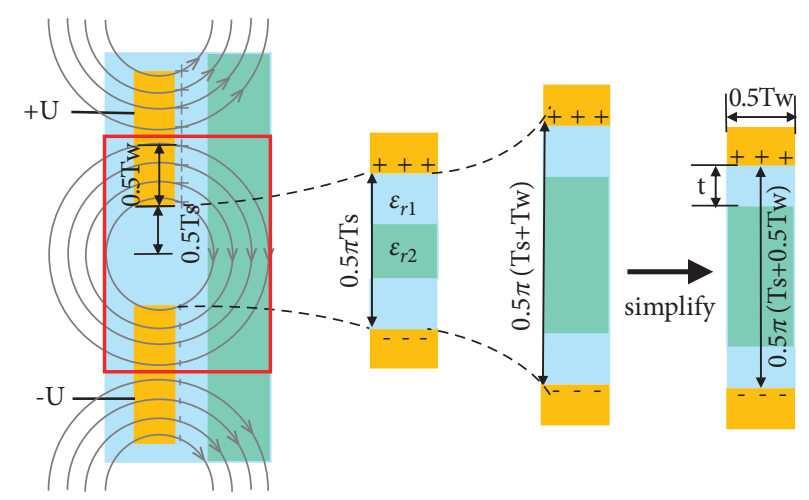

FIGURE 2: Electrostatic adhesion principle of the nonconductor.

Additionally, the electroadhesive force per area can be calculated by substituting equation (9) as

$$
f_{s}=\frac{F_{z}}{L_{M} * L_{N}},
$$

where $L_{M} * L_{N}$ is the whole area of the pad.

\section{Optimization Design Strategy}

The main objective of the optimization design strategy is to design an electroadhesive pad that achieves maximum force for a specific application by comprehensively considering realistic factors and influencing parameters. The maximum force refers to the maximum electroadhesive force that can be provided by the pad to the target object under ideal conditions with the exclusion of external environmental influencing factors. The ideal conditions are set as follows:

(1) The dielectric materials are linear, homogeneous, and isotropic.

(2) The surface of the electroadhesive pad is completely flat and without an air gap between the pad and the object.
(3) The contact area with the target object must be equal to the area of the pad; that is, the area of the pad and the object are assumed to be the same.

(4) The thickness of the object must be greater than the maximum radius of the electric field to obtain $100 \%$ of the effective force.

From the multiparameter theoretical model, we can find that there are five key parameters $\left(U, t, \varepsilon_{r 1}, T_{w}\right.$, and $\left.T_{s}\right)$ that directly affect the sum electroadhesive force and the force increases with the applied voltage, the relative dielectric constant of the material, and the size of the electroadhesive pad and decreases with the thickness of the insulation layer. On another aspect, these parameters are limited by practical factors, such as the constraints of device, material, and fabrication. Therefore, by considering the relationships between the electroadhesive force and these five parameters and the limitations, an optimization design strategy was developed based on the electroadhesive force generation mechanism as shown in Figure 3.

Following this strategy, the range of dimensions of the object being processed is firstly considered to determine the appropriate value of the pad size. After the dimensional parameters $L_{M}$ and $L_{N}$ are determined, the next step is to 


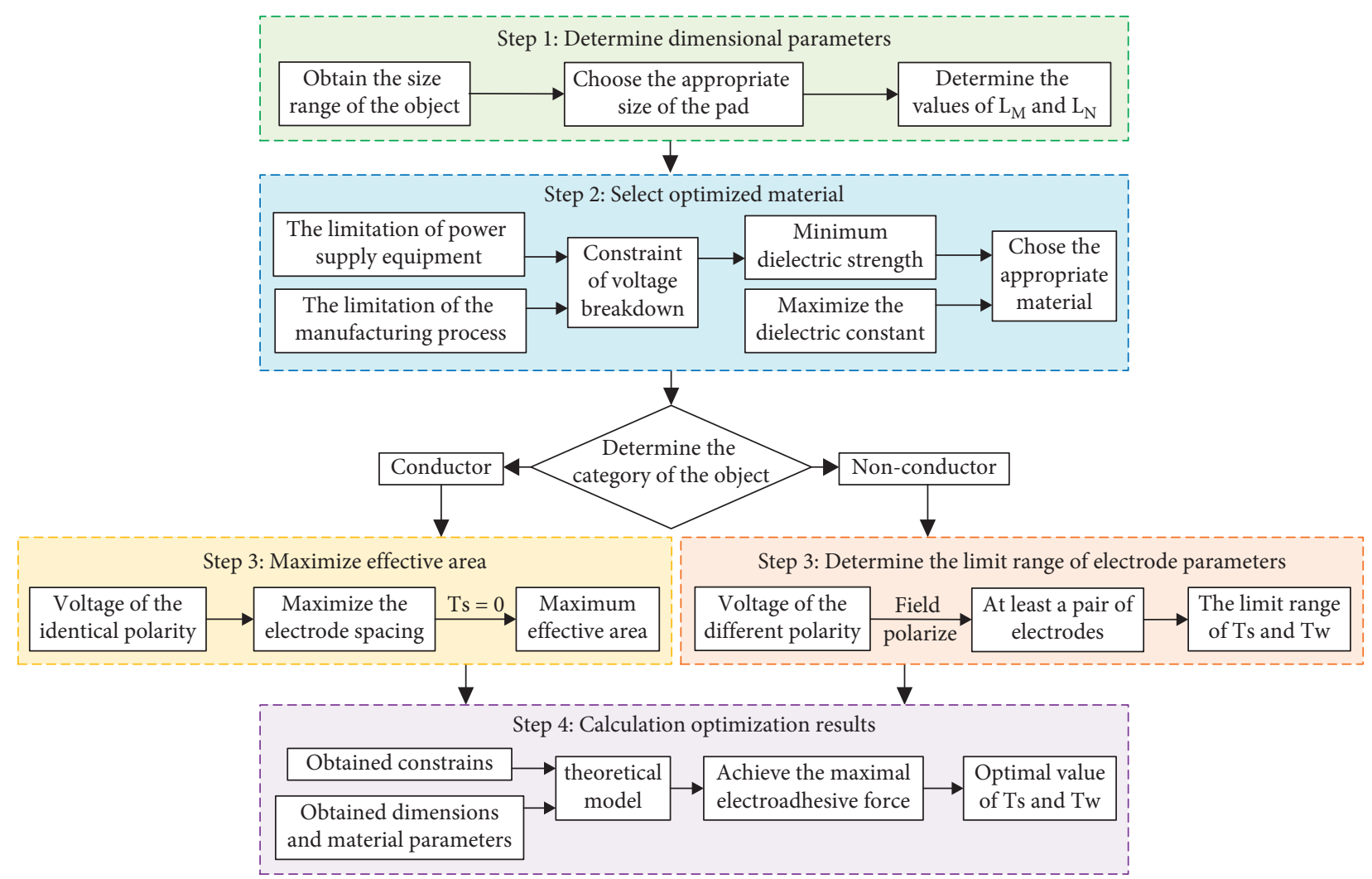

FIGURE 3: The optimization design strategy.

determine the optimal value of the insulation material parameters $t$ and $\varepsilon_{r 1}$.

Considering the limitations of the power supply equipment (maximum value of voltage that can be supplied) and the manufacturing method (minimum level of process that can be achieved), the minimum value of the dielectric strength of the material can be deduced according to the principle of dielectric breakdown. Accordingly, a suitable dielectric material is selected that has the maximum relative dielectric constant while ensuring no breakdown (dielectric strength greater than the minimum dielectric strength). Then, judge whether the object is a conductor or a nonconductor.

For the conductor, according to the mechanism of electrostatic induction, a voltage of the same polarity can be applied to the electrodes. Hence, the problem of electric breakdown between the electrodes is not required, the electrode spacing is not limited by electric breakdown anymore, and the minimum electrode spacing $T_{s}$ can be 0 . From equation (1), the electroadhesive force can be increased by increasing the area of the electrodes, so the value of the electrode spacing is set to the minimum value to obtain the maximum area. Then, the optimal electrode width is equal to the length of the pad and the maximum effective area is calculated to be $L_{M} * L_{N}$ by substituting equation (2), which is also equal to the total area of the electroadhesive pad.

For the nonconductor, the third step is to determine the range of values for the width and spacing of the electrodes. According to the mechanism of dielectric polarization, to obtain an electric field that produces polarization, a voltage of opposite polarity must be applied to the adjacent electrodes and the pad is required to include at least one pair of electrodes or at least one unit. Furthermore, several practical limitations are taken into account, such as the limitation of the maximum voltage of the power supply equipment, the limitation of the manufacturing method on the minimum electrode width and spacing, as well as the thinnest insulation layer thickness, the limitation of the overall dimensions on the maximum electrode width and spacing, and the limitation of the breakdown voltage on the dielectric strength and the thickness of the insulation layer as well as the electrode spacing. By substituting the known parameters, the limits on the range of electrode parameters can be derived from the following inequalities: 


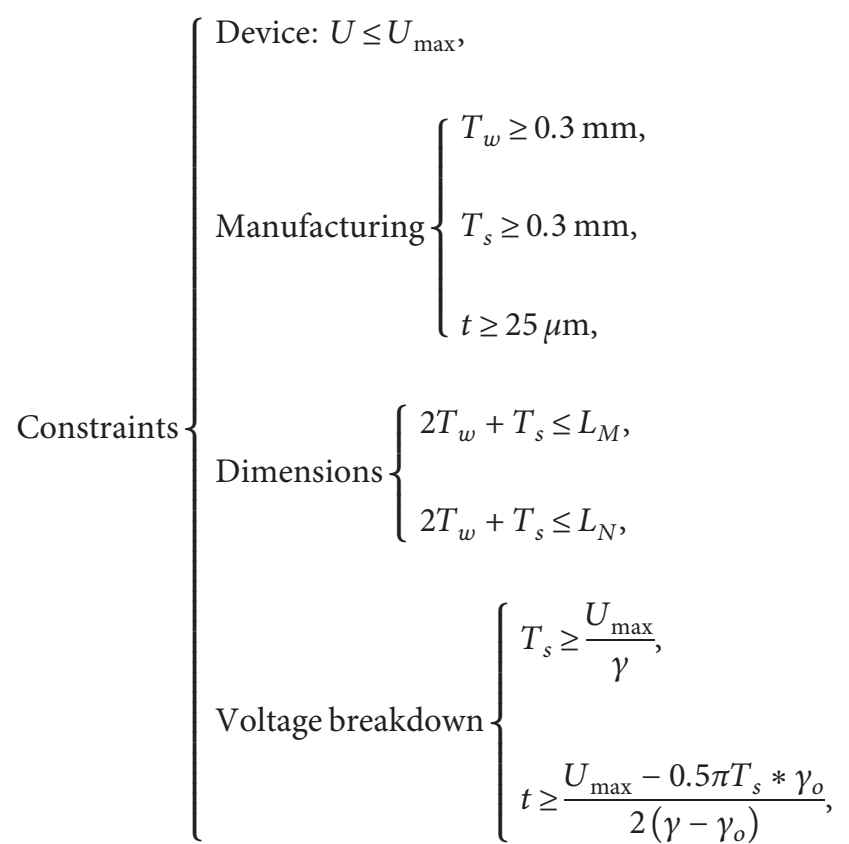

where $\gamma$ is the dielectric strength of the dielectric material and $\gamma_{o}$ is the dielectric strength of air. Additionally, based on the equivalent capacitance model for the nonconductor, under the condition of ensuring that no voltage breakdown occurs even in the absence of the object, the limitation on the dielectric breakdown is the inequality containing $t$ and can be expressed as

$$
2 t \gamma+\left(0.5 \pi T_{s}-2 t\right) * \gamma_{o} \geq U_{\max }
$$

Finally, by substituting the obtained values of the relative permittivity of the material and the size of the electroadhesive pad into the theoretical model, the optimal values of the electrode spacing and width that maximize the force under the constraints can be found according to the relationship between the remaining parameters in the theoretical model and the electroadhesive force (the force increases with increasing voltage and decreases with increasing thickness of the insulation layer).

\section{Optimization Analysis and Results}

4.1. Influencing Factors of the Optimal Electrode Width. According to the theoretical model, the force generated by the electroadhesive pad can be maximized by adjusting the parameters of the electrode structure. For conductive objects, the optimal electrode width $T_{w}$ is equal to the length of the pad when the electrode spacing $T_{s}=0$. For the nonconductor, according to the theoretical relationship between the electroadhesive force and each parameter, the optimal value of each parameter can be obtained by considering the restricted range of values for each parameter. The optimal voltage $U$ is obtained by taking the maximum value, and the optimal thickness $t$ and electrode spacing $T_{s}$ are obtained by taking the minimum value, while the value of the optimal electrode width $T_{w}$ seems to be arbitrary, since the optimal $T_{w}$ may be different when some parameters are changed. In order to further investigate the factors affecting the optimal value of electrode width, multiple control tests were conducted. The initial values were set as $T_{s}=0.3 \mathrm{~mm}$, $L_{M}=160 \mathrm{~mm}, L_{N}=80 \mathrm{~mm}, U=5000 \mathrm{~V}, t=0.04 \mathrm{~mm}$; then, based on this, the values of each parameter were changed, their corresponding optimal values of electrode widths were calculated by the theoretical model, and the results are presented in Figure 4.

As can be seen from the results in Figure 4(a), the value of the optimal $T_{w}$ increases with the increase of electrode spacing and it is also worth noting that the trend of the effect of $T_{s}$ on $T_{w}$ is similar to the trend of the effect on force. In addition, the output results of different thicknesses of the insulation layer are shown in Figure 4(b) and it can be found that the optimal $T_{w}$ increases with the increase of parameter $t$. The variation curve is similar to that of $t$ versus the adhesive force and the degree of increase is smaller than that of $T_{s}$. Moreover, considering the actual situation, the value of the thickness of the insulating layer is always much smaller than the value of the electrode width, which means that the actual change in the thickness has little impact on the optimal electrode width. As shown in Figure 4(c), $T_{w}$ decreases with the increase of the relative dielectric constant $\varepsilon_{r 1}$ of the insulating material, and the degree of reduction also becomes smaller. However, compared to the relationship between $\varepsilon_{r 1}$ and $T_{w}$, the relative permittivity $\varepsilon_{r 2}$ of the target object in Figure $4(\mathrm{~d})$ has an opposite relationship. $T_{w}$ increases as $\varepsilon_{r 2}$ increases and obviously the change in $\varepsilon_{r 2}$ has a greater effect on the electroadhesive force than that of $\varepsilon_{r 1}$, implying that the change of the force is more sensitive to $\varepsilon_{r 2}$. Furthermore, it can be seen from Figures 4(e) and 4(f) that the voltage and the width of the electroadhesive pad have no effect on the optimal $T_{w}$. According to the analysis of the theoretical model, this is because they are directly proportional to the total force.

According to the total force theoretical model, it should be noted that the size of $L_{N}$ also has an effect on the optimal electrode width. As shown in Figure 5(a), with the increase of $L_{N}$, the optimal $T_{w}$ value also increases, and the red line indicates the trajectory of the change of $T_{w}$. As shown in Figure 5(b), the symbol $\Delta T_{w}$ represents the change amount of the optimal $T_{w}$ relative to the minimum value with the change of $L_{N}$, which can be obtained by $T_{w}-T_{w\left(L_{N}=20\right)}$. Additionally, we found that when the difference between $L_{N}$ and $T_{w}$ becomes larger, $\Delta T_{w}$ becomes smaller, which means that the influence of $L_{N}$ becomes smaller.

From the above results, it can be observed that different electrode widths, dielectric layer thicknesses, relative dielectric constants, and electroadhesive pad lengths all affect the optimal electrode width, and the influence trend is also related to the relationship between each parameter and the electroadhesive force. These influences validate that the optimal $T_{w}$ values are different for application objects with different sizes and materials. Therefore, it is necessary to propose a strategy for designing electroadhesive pads for specific applications. 


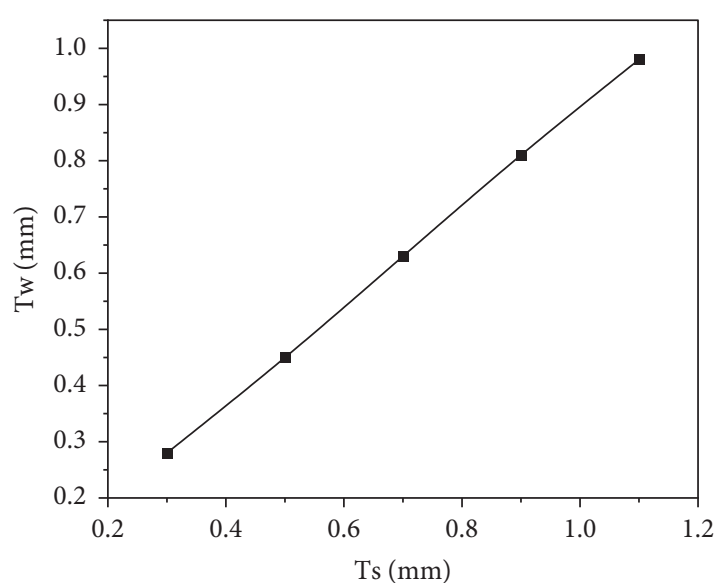

(a)

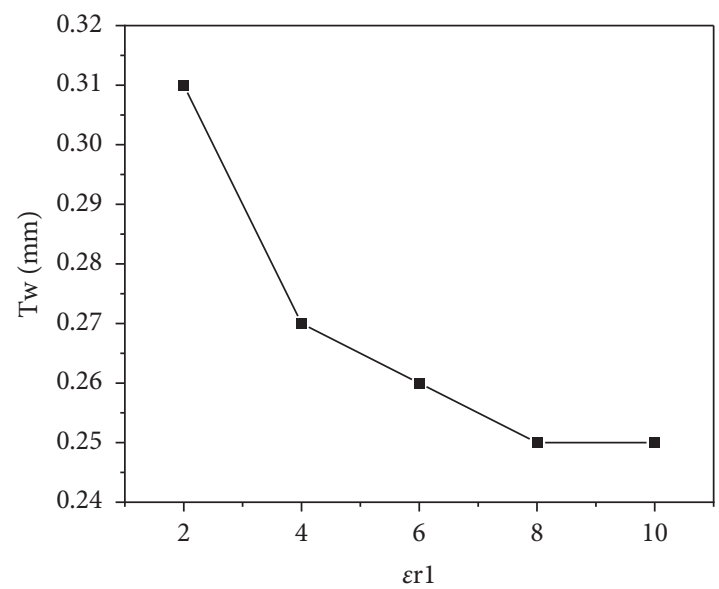

(c)

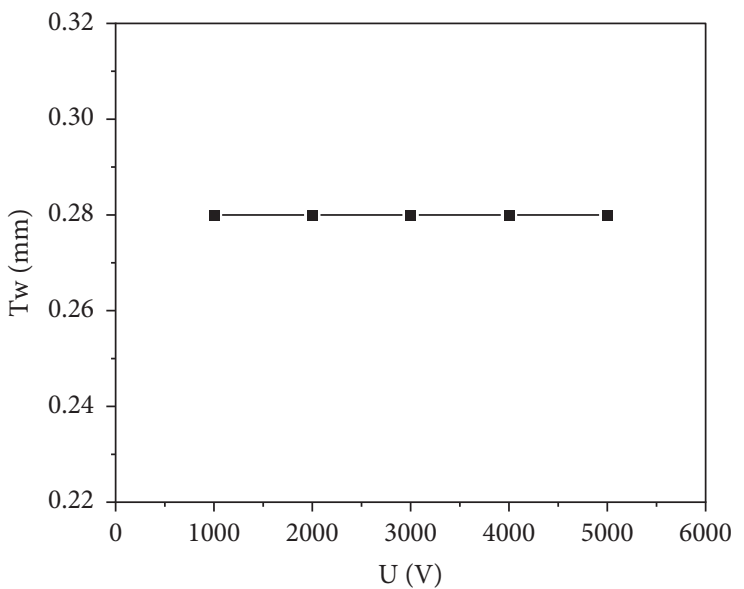

(e)

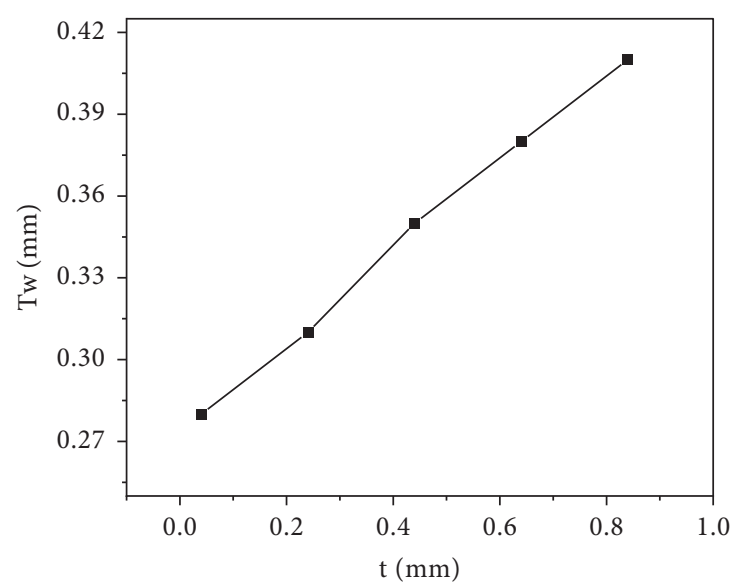

(b)

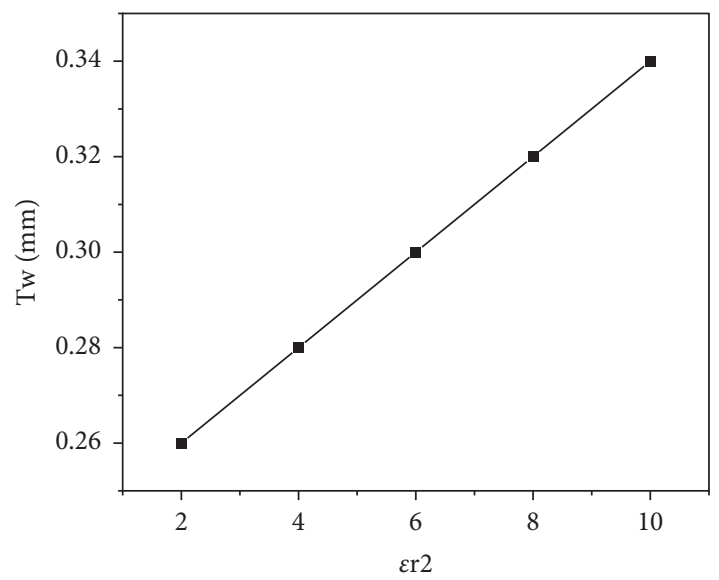

(d)

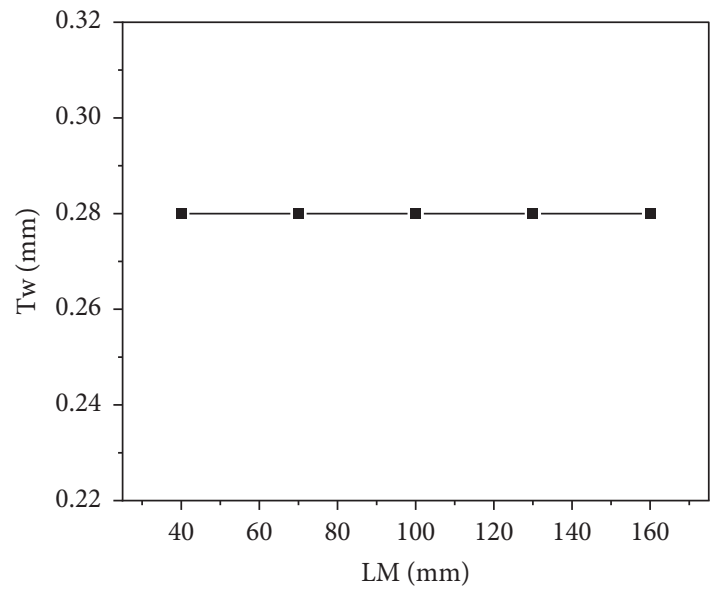

(f)

Figure 4: The relationship between the optimal $T_{w}$ and (a) the electrode spacing, (b) the dielectric layer thickness, (c) the relative permittivity of dielectric material, (d) the relative permittivity of objects, (e) the applied voltage, and (f) the pad length.

4.2. A Worked Example. The electroadhesion technology was developed from the beginning to solve the problems of product surface damage and complex configuration of different products caused by traditional mechanical clamping equipment in the semiconductor and flat panel industries. The structural design of the electroadhesive pad is critical to the magnitude and stability of the adhesive force provided by the electroadhesion system. To test the effect of the optimization design strategy on the performance improvement of the electroadhesion system, the application of cell phone screen protection film in the panel industry is taken as an example. 


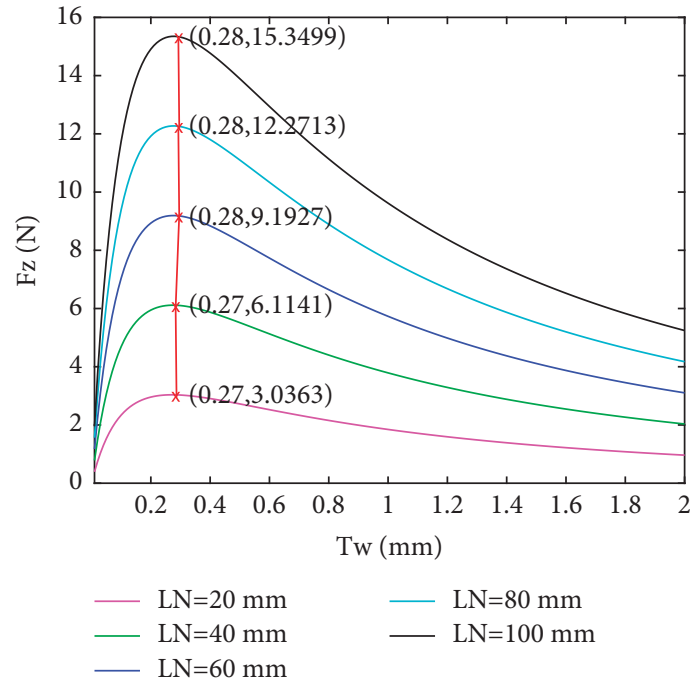

(a)

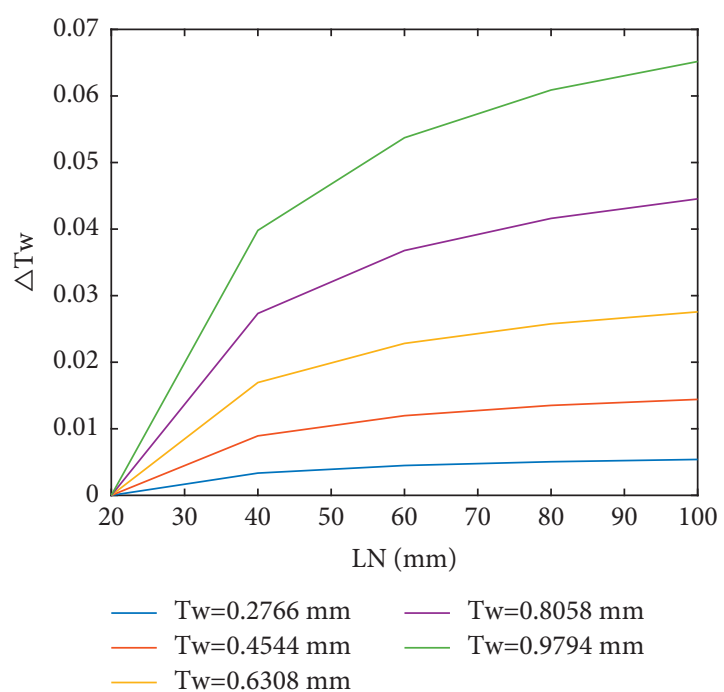

(b)

Figure 5: The relationship between the optimal $T_{w}$ and the pad width. (a) The influence of different $L_{N}$. (b) The change amount of the optimal $T_{w}$ with the change of $L_{N}$.

According to the proposed strategy, firstly, we obtain information about the size range of the protective film of the mobile phone screen: the width range is from $60 \mathrm{~mm}$ to $80 \mathrm{~mm}$ and the length range is from $120 \mathrm{~mm}$ to $160 \mathrm{~mm}$. In order to ensure that the force on the protective film is uniform; that is, the force area is equal to the area of the protective film, the size of the pad is determined as $L_{M}=160 \mathrm{~mm}, L_{N}=80 \mathrm{~mm}$. The second step is to select an appropriate insulating material. Considering the maximum voltage of the $\mathrm{DC}$ power supply is $5 \mathrm{kV}$, the minimum dielectric layer thickness, electrode width, and spacing that can be manufactured are $0.025 \mathrm{~mm}, 0.3 \mathrm{~mm}$, and $0.3 \mathrm{~mm}$, respectively. According to the constraints of the breakdown voltage in equation (11), the minimum dielectric strength of the material can be calculated as $\gamma=81.3 \mathrm{kV} / \mathrm{mm}$. Considering the requirements of avoiding breakdown and maximizing $\varepsilon_{r 1}$, a PI material with a relative dielectric constant of 3.4 and a dielectric strength of $\gamma=125 \mathrm{kV} / \mathrm{mm}$ was chosen. Then, since the screen is made of glass, it is judged to be nonconductor. Following the design strategy, in the next step, the variation range of the electrode structure parameters can be obtained by substituting the dimensional and material parameters into equation (11) and calculating; then, the limit conditions of these parameters can be expressed as $U \leq 5 \mathrm{kV}, t \geq 40 \mu \mathrm{m}, 0.3 \mathrm{~mm} \leq T_{w} \leq 39.85 \mathrm{~mm}$, $0.3 \mathrm{~mm} \leq T_{s} \leq 79.6 \mathrm{~mm}$. Finally, according to the theoretical model, the fminsearchbnd() function of MATLAB is used to calculate the optimal values of the remaining parameters by inputting the known parameters and constraints. The result is given as follows: the maximum attraction force is $12.2490 \mathrm{~N}$ (per area force is $0.00096 \mathrm{~N} / \mathrm{mm}^{2}$ or $0.96 \mathrm{kpa}$ ) under the condition of $U=5 \mathrm{kV}, T_{w}=0.3 \mathrm{~mm}$ (the theoretical optimal value is $0.2766 \mathrm{~mm}), T_{s}=0.3 \mathrm{~mm}$, $t=0.04 \mathrm{~mm}, \varepsilon_{r 1}=3.4$, and $\varepsilon_{r 2}=4.1$.

From the results, it can be concluded that the smaller the spacing between the electrodes and the thickness of the dielectric, the greater the applied voltage and the greater the electroadhesive force that can be obtained when designing this electroadhesive pad. Moreover, the total maximum electroadhesive force can be achieved at an optimal electrode width of $0.3 \mathrm{~mm}$. Furthermore, with better equipment and more advanced manufacturing methods, the limits of each parameter can be further extended and the maximum force can therefore be higher. Finally, under the premise of guaranteeing stable adhesion to the target, the structure, material, and size of the electroadhesive pad can also be adjusted according to the individual needs of the user.

4.3. Simulation Results. To have a better understanding of the mechanism of electroadhesion and to verify the accuracy of the above theoretical results, a 3D simulation model has been created in COMSOL. The model can be employed to simulate the magnitude and distribution of the potential and electric field generated by the applied voltage, as well as the polarized charge density generated by the dielectric polarization, and to calculate the value of the resulting electroadhesive force. As shown in Figure 6(a), the 3D simulation model consists of an electrode pattern, dielectric layer, and target object, and the relevant structural parameter settings are also included Figure 6(a).

For the simulation of the electroadhesion, the following settings were made in order to meet our goal of outputting the maximum force under ideal conditions: the materials used are linear, homogeneous, and isotropic; the surfaces of the layers of the structure are completely flat; the electroadhesive pad is in close contact with the object without air gap; the areas of the electroadhesive pad and the object are assumed to be the same; and the thickness of the object is set to be greater than the maximum radius of the electric field. Besides, according to the results obtained from the optimization strategy, the properties of each component are 


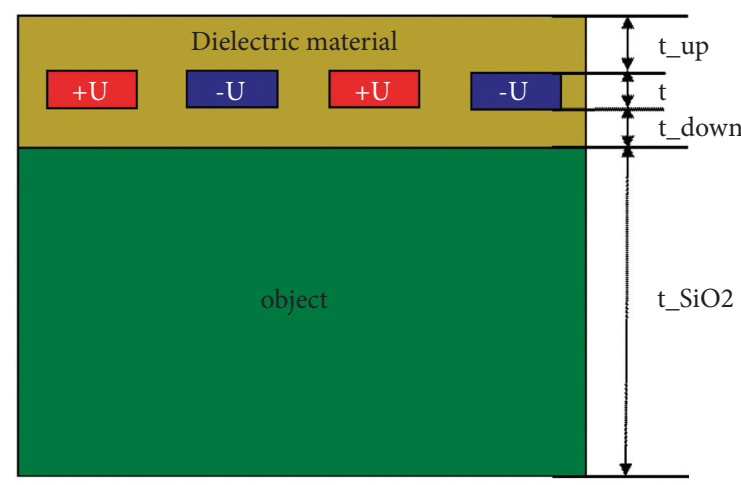

(a)

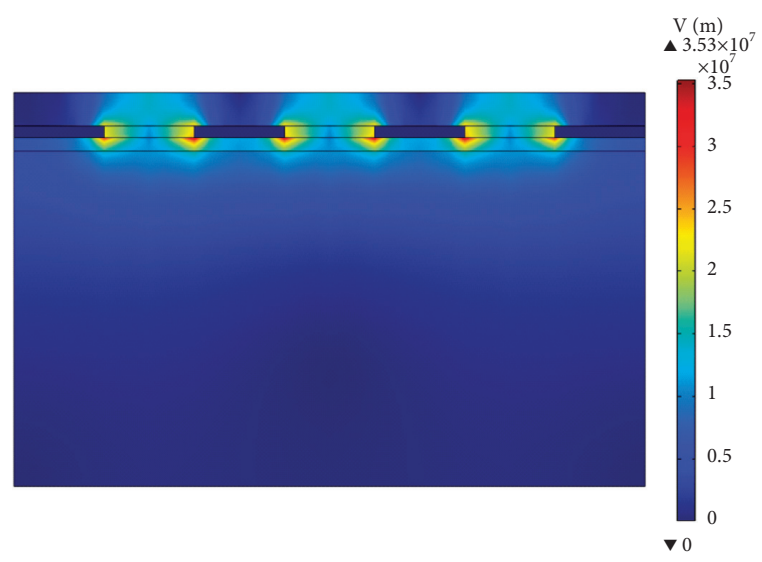

(c)

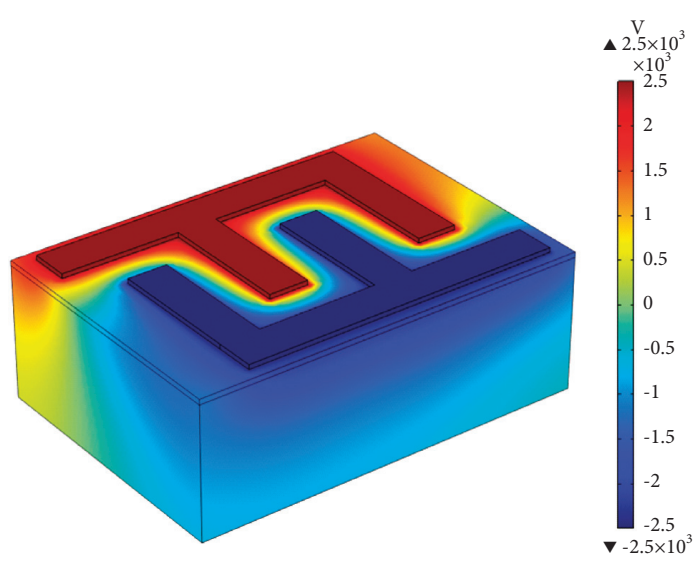

(b)

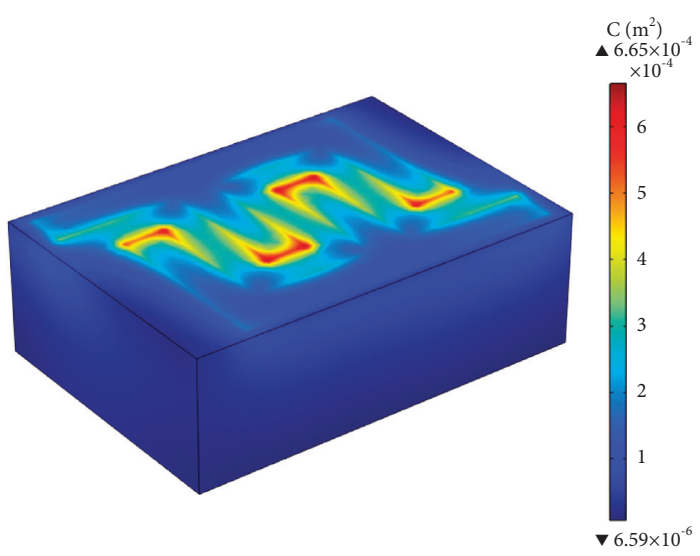

(d)

Figure 6: (a) Structure of 3D simulation model; the magnitude and distribution of (b) the potential, (c) the electric field, and (d) the polarization charge density.

added, including the type of material (the electrodes are pure copper with perfect conductivity), the relative permittivity ( $\varepsilon_{r 2}=4.2$ for the object (glass), $\varepsilon_{a}=1.0$ for air, and $\varepsilon_{r 1}=$ 3.4 for the insulating layer (PI)), and the corresponding voltage $(U=5 \mathrm{kv})$ and the structural parameters in Table 1 .

In addition, due to the limitation of the software, the simulation takes a long time and requires a lot of computer resources when the model is large. Therefore, to speed up the simulation and reduce the computer burden, we chose two units $(n=2)$ for the simulation.

According to the color legend on the right, as shown in Figure 6(b), it can be seen that voltages of equal magnitude and opposite polarity $( \pm 2.5 \mathrm{kv})$ are applied to the two electrodes, and the potential distribution corresponding to the color distribution can also be viewed. In addition, the magnitude and distribution of the electric field are displayed in Figure 6(c). It can be noticed that further away from the electrode, the higher the electric field intensity, which is agreed with the analytical results of the concentric electric field model. As shown in Figure 6(d), the polarization charge density is distributed along the shape of the electrodes. The maximum value of the density appears near the end of the electrode strips, indicating that the polarization effect in these regions is the strongest. These simulation results are similar to those in the work performed by Bigharaz et al. [23], Kim et al. [24].

To validate the accuracy of the theoretical model, several sets of control simulations were set up in COMSOL by changing the values of electrode spacing. Based on the setup of the simulation model, to more easily compare the theoretical and simulation results, the total electroadhesive force obtained from the simulation was divided by the area of the simulated pad to obtain the force value per unit area. The theoretical force value per unit area under the same conditions was also calculated using equation (10), and the simulation results are shown in Figure 7.

As shown in Figures 7(a)-7(e), the electroadhesive force per unit area varies with $T_{w}$ and has a maximum value. The red points in Figures 7(a)-7(e) correspond to the maximum force values, and the red texts denote the coordinates of the points corresponding to the optimal $T_{w}$ and the maximum force values. From the results in these plots, it can be found that the optimal $T_{w}$ values corresponding to different $T_{s}$ are different and the optimal $T_{w}$ values of the simulation are around $0.1 \mathrm{~mm}$, which is very small compared to the theoretical results in Figure 4(a). From Figure 7(f), the trend of the simulation results matches the trend of the theoretical results but there are some numerical differences. The reasons 
TABLE 1: Structural parameters of the simulation model.

\begin{tabular}{lccc}
\hline Name & Expression & Value & Description \\
\hline$t$ & $35(\mathrm{um})$ & $3.5 \mathrm{E}-5(\mathrm{~m})$ & Electrode thickness \\
$w$ & $0.5(\mathrm{~mm})$ & $5 \mathrm{E}-4(\mathrm{~m})$ & Electrode width \\
$g$ & $0.5(\mathrm{~mm})$ & $5 \mathrm{E}-4(\mathrm{~m})$ & Electrode spacing \\
$n$ & 2 & 2 & Electrode pairs \\
$l$ & $72(\mathrm{~mm})$ & $0.072(\mathrm{~m})$ & Electrode layer width \\
$L$ & $(g+w) * 2 * \mathrm{n}$ & $0.004(\mathrm{~m})$ & Electrode layer length \\
L1 & $(L+g) * 1.1$ & $0.00495(\mathrm{~m})$ & Pad length \\
W1 & $(l+2 * w+g) * 1.1$ & $0.08085(\mathrm{~m})$ & Pad width \\
t_down & $40(\mathrm{um})$ & $4 \mathrm{E}-5(\mathrm{~m})$ & Lower dielectric layer thickness \\
t_up & $100(\mathrm{um})$ & $1 \mathrm{E}-4(\mathrm{~m})$ & Upper dielectric layer thickness \\
t_SiO2 & $1(\mathrm{~mm})$ & $0.001(\mathrm{~m})$ & Object thickness \\
Air_d & $2(\mathrm{~mm})$ & $0.002(\mathrm{~m})$ & Atmospheric spatial expansion \\
\hline
\end{tabular}

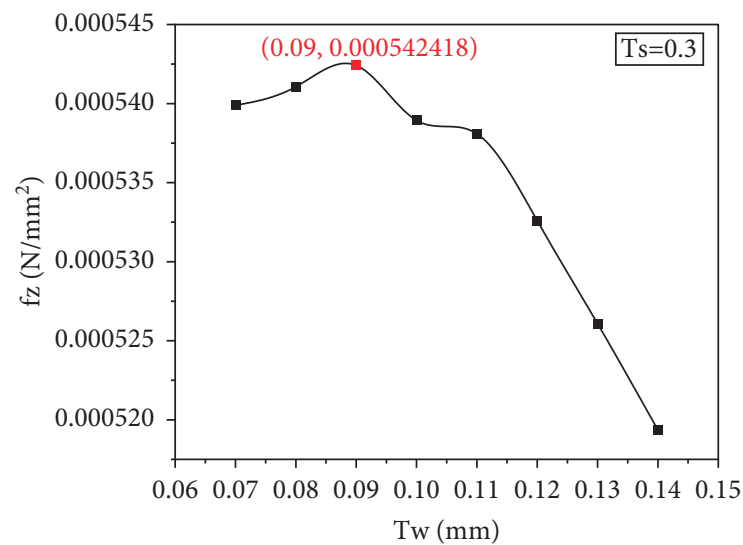

(a)

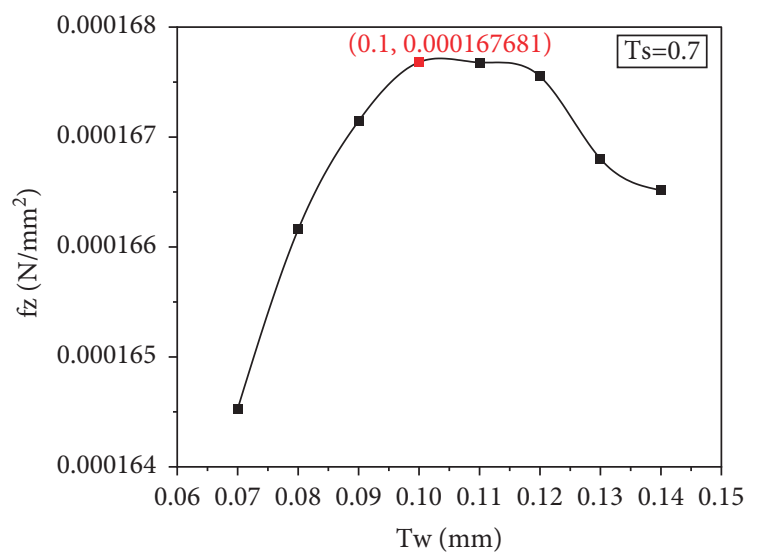

(c)

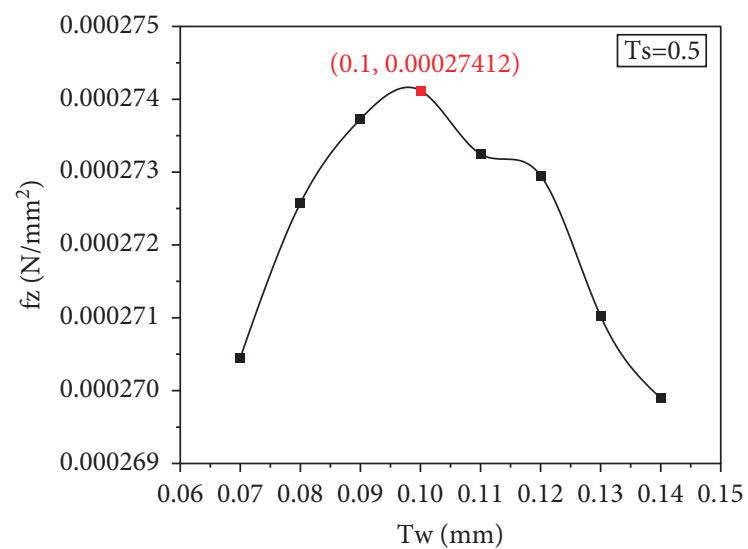

(b)

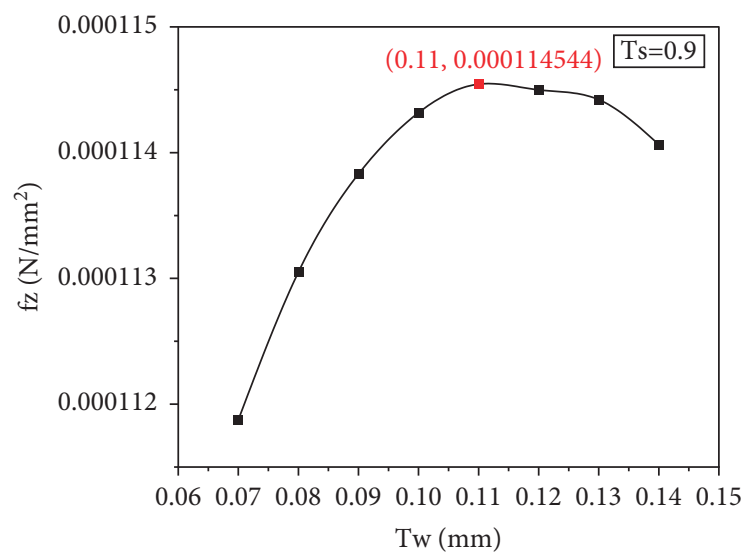

(d)

Figure 7: Continued. 


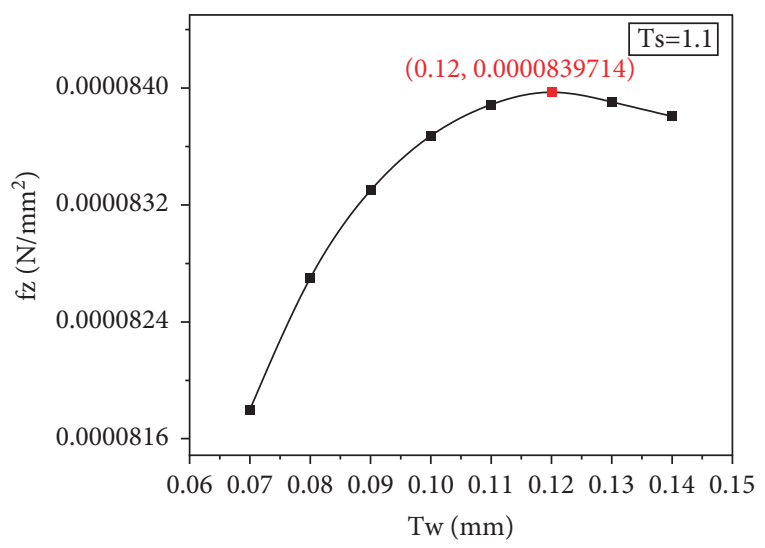

(e)

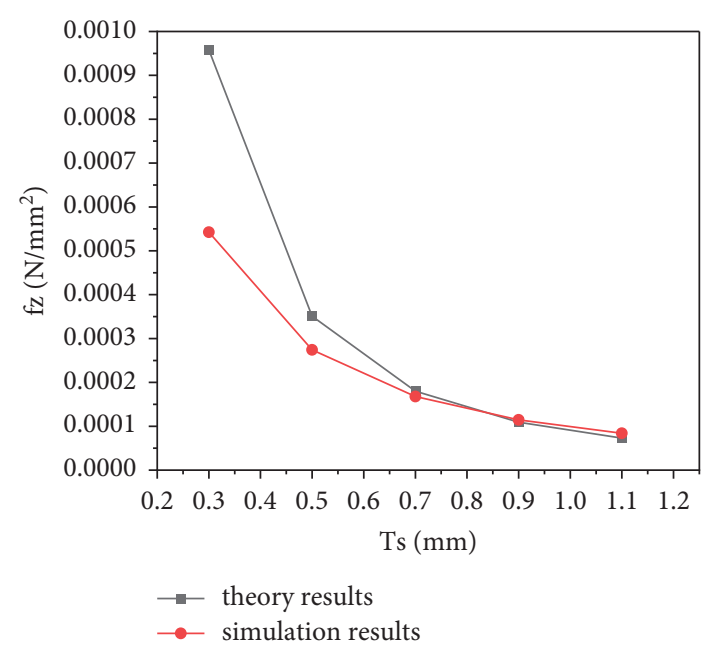

(f)

Figure 7: (a-e) Simulation results of the optimal $T_{w}$ under different $T_{s}$. (f) Comparison of simulated and theoretical values.

for the numerical differences between the theoretical and simulation results were analyzed as follows: on the one hand, this may be due to the simplified equivalent treatment we have done in the theoretical model, such as simplifying the elliptical electric field between the electrodes to the concentric electric field, taking the middle value of the capacitance spacing $T_{s}+0.5 T_{w}$, and so forth. On the other hand, it may be caused by the simulation settings. For example, only 2 units were simulated and the actual number of units was different from this, which may cause errors. Moreover, the predefined mesh size is set directly to a regular value for convenience. This may also be the reason for the differences since the size of the grid refinement can also affect the accuracy of the results.

\section{Conclusion}

In this paper, a multiparameter theoretical analysis model containing dimensional, structural, and material parameters has been developed by using the parallel plate capacitance model, and an application-specific design strategy has been proposed by considering key design parameters and practical influencing factors. The strategy is dedicated to designing electroadhesive pads with optimal performance and easy implementation. In addition, the influence of each parameter on the optimization results has been investigated, and an optimized structure has been designed for an electroadhesive pad application example. Finally, the accuracy of the optimization results and the feasibility of the optimization strategy have been further proved using a 3D simulation model. The results have validated that the dimensional and material parameters of the application object affect the optimization design of the corresponding electroadhesive pad, and the practical constraints of the key parameters also affect the final optimization results. By employing our multiparameter optimization design strategy, optimization results with high accuracy and suitable for practical applications can be obtained. This study not only will be useful for the structural optimization and performance enhancement of electroadhesive devices in various applications but also will provide a certain reference for the study of electroadhesion mechanisms.

\section{Data Availability}

The data will be available from the corresponding author upon request.

\section{Conflicts of Interest}

The authors declare that they have no conflicts of interest.

\section{Acknowledgments}

This work was supported by the National Natural Science Foundation of China (Project no. 31901415), the Natural Science Foundation of Jiangsu Province (Grant no. BK20180515), and the Jiangsu Science and Technology Innovation Fund (JASTIF) (Grant no.CX(21)3146).

\section{References}

[1] A. Johnsen and K. Rahbek, "A physical phenomenon and its applications to telegraphy, telephony, etc," Journal of the Institution of Electrical Engineers, vol. 61, no. 320, pp. 713725, 2010.

[2] J. Germann, B. Schubert, and D. Floreano, "Stretchable electroadhesion for soft robots," in Proceedings of the 2014 IEEE/RSJ International Conference on Intelligent Robots and Systems, IEEE, Chicago, IL, USA, September 2014.

[3] D. Ruffatto, A. Parness, and M. Spenko, "Improving controllable adhesion on both rough and smooth surfaces with a hybrid electrostatic/gecko-like adhesive," Journal of the Royal Society Interface, vol. 11, no. 93, Article ID 20131089, 2014.

[4] J. Guo, K. Elgeneidy, C. Xiang, N. Lohse, L. Justham, and J. Rossiter, "Soft pneumatic grippers embedded with stretchable electroadhesion," Smart Material Structures, vol. 27, no. 5, 2018. 
[5] E. W. Schaler, D. Ruffatto, P. Glick, V. White, and A. Parness, "An electrostatic gripper for flexible objects," in Proceedings of the 2017 IEEE/RSJ International Conference on Intelligent Robots and Systems (IROS), September 2017.

[6] V. Cacucciolo, J. Shintake, and H. Shea, "Delicate yet strong: characterizing the electro-adhesion lifting force with a soft gripper," in Proceedings of the 2019 2nd IEEE International Conference on Soft Robotics (RoboSoft), April 2019.

[7] J. Guo, J. Leng, and J. Rossiter, "Electroadhesion technologies for robotics: a comprehensive review," IEEE Transactions on Robotics, vol. 36, no. 2, pp. 313-327, 2020.

[8] K. Asano, F. Hatakeyama, and K. Yatsuzuka, "Fundamental study of an electrostatic chuck for silicon wafer handling," IEEE Transactions on Industry Applications, vol. 38, no. 3, pp. 840-845, 2002.

[9] P. M. Taylor, G. J. Monkman, and G. E. Taylor, "Electrostatic grippers for fabric handling," in Proceedings of the IEEE International Conference on Robotics \& Automation, IEEE, Philadelphia, PA, USA, April 1988.

[10] B. Sun and X. Zhang, "A new electrostatic gripper for flexible handling of fabrics in automated garment manufacturing," in Proceedings of the 2019 IEEE 15th International Conference on Automation Science and Engineering (CASE), IEEE, Vancouver, Canada, August 2019.

[11] H. Prahlad, R. Pelrine, S. Stanford, J. Marlow, and R. Kornbluh, "Electroadhesive robots - wall climbing robots enabled by a novel, robust, and electrically controllable adhesion technology," in Proceedings of the IEEE International Conference on Robotics \& Automation, IEEE, Pasadena, CA, USA, May 2008.

[12] A. Yamamoto, T. Nakashima, and T. Higuchi, "Wall climbing mechanisms using electrostatic attraction generated by flexible electrodes," in Proceedings of the International Symposium on Micro-nanomechatronics \& Human Science, November 2007.

[13] C. Rui, L. Rong, J. Chen, and Z. Jin, “A gecko inspired wallclimbing robot based on electrostatic adhesion mechanism," in Proceedings of the IEEE International Conference on Robotics \& Biomimetics, IEEE, Shenzhen, China, December 2013.

[14] G. Gu, J. Zou, R. Zhao, X. Zhao, and X. Zhu, "Soft wallclimbing robots," Science Robotics, vol. 3, no. 25, Article ID eaat2874, 2018.

[15] S. A. Suresh, D. L. Christensen, E. W. Hawkes, and M. Cutkosky, "Surface and shape deposition manufacturing for the fabrication of a curved surface gripper," Journal of Mechanisms \& Robotics, vol. 7, no. 2, Article ID 021005, 2015.

[16] M. Dadkhah, Z. Zhao, N. Wettels, and M. Spenko, "A selfaligning gripper using an electrostatic/gecko-like adhesive," in Proceedings of the IEEE/RSJ International Conference on Intelligent Robots \& Systems, October 2016.

[17] V. Alizadehyazdi, M. Bonthron, and M. Spenko, "An electrostatic/gecko-inspired adhesives soft robotic gripper," IEEE Robotics and Automation Letters, vol. 5, pp. 4679-4686, 2020.

[18] K. Tanaka and M. Spenko, "A gecko-like/electrostatic gripper for free-flying perching robots," in Proceedings of the 2020 IEEE Aerospace Conference, IEEE, Big Sky, MT, USA, March 2020.

[19] J. Guo, T. Bamber, Y. Zhao, M. Chamberlain, L. Justham, and M. Jackson, "Toward adaptive and intelligent electroadhesives for robotic material handling," IEEE Robotics and Automation Letters, vol. 2, no. 2, pp. 538-545, 2017.
[20] N. Bo and J. Guo, "Electroadhesion for soft adhesive pads and robotics: theory and numerical results," Soft Matter, vol. 15, 2019.

[21] J. Guo, T. Bamber, and M. R. Chamberlain, "Optimization and experimental verification of coplanar interdigital electroadhesives," Journal of Physics D Applied Physics, vol. 49, no. 41, Article ID 415304, 2016.

[22] S. M. J. Mahmoudzadeh Akherat, M. A. Karimi, V. Alizadehyazdi, S. Asalzadeh, and M. Spenko, "A tunable dielectric to improve electrostatic adhesion in electrostatic/ microstructured adhesives," Journal of Electrostatics, vol. 97, pp. 58-70, 2019.

[23] M. Bigharaz, T. Schenkel, and P. A. Bingham, "Increasing force generation in electroadhesive devices through modelling of novel electrode geometries," Journal of Electrostatics, vol. 109, 2021.

[24] J. H. Kim, S. H. Kang, and S. Cho, "Shape design optimization of interdigitated electrodes for maximal electro-adhesion forces," Structural and Multidisciplinary Optimization, vol. 61, no. 5, pp. 1843-1855, 2020.

[25] K. Choi, Y. Chan Kim, H. Sun et al., "Quantitative electrode design modeling of an electroadhesive lifting device based on the localized charge distribution and interfacial polarization of different objects," ACS Omega, vol. 4, no. 5, pp. 7994-8000, 2019.

[26] J. D. West, J. Mici, J. F. Jaquith, and H. Lipson, "Design and optimization of millimeter-scale electroadhesive grippers," Journal of Physics D Applied Physics, vol. 53, no. 43, 2020.

[27] W. Wang, D. Fan, R. Zhu, P. Wang, and H. Wang, "Modeling and optimization of electrostatic film actuators based on the method of moments," Soft Robotics, 2020, In press.

[28] J. P. Díaz Téllez, J. Krahn, and C. Menon, "Characterization of electro-adhesives for robotic applications," in Proceedings of the 2011 IEEE International Conference on Robotics and Biomimetics, IEEE, Karon Beach, Thailand, December 2011.

[29] J. Guo, M. Tailor, T. Bamber, M. Chamberlain, L. Justham, and M. Jackson, "Investigation of relationship between interfacial electroadhesive force and surface texture," Journal of Physics D: Applied Physics, vol. 49, no. 3, Article ID 035303, 2016. 